\title{
Essais
}

Revue interdisciplinaire d'Humanités

17 | 2021

Quels lieux pour les morts?

\section{Quel avenir pour les cimetières à Shanghai ?}

What Future for Cemeteries in Shanghai?

\section{Maylis Bellocq}

\section{(2) OpenEdition}

1 Journals

\section{Édition électronique}

URL : https://journals.openedition.org/essais/8580

DOI : $10.4000 /$ essais. 8580

ISSN : 2276-0970

Éditeur

École doctorale Montaigne Humanités

\section{Édition imprimée}

Date de publication : 15 avril 2021

Pagination : 61-73

ISBN : 979-10-97024-09-3

ISSN : 2417-4211

Référence électronique

Maylis Bellocq, "Quel avenir pour les cimetières à Shanghai ? », Essais [En ligne], 17 | 2021, mis en ligne le 27 avril 2021, consulté le 18 janvier 2023. URL : http://journals.openedition.org/essais/8580 ; DOI : https://doi.org/10.4000/essais.8580 


\section{Quel avenir pour les cimetières à Shanghai ?}

\section{Maylis Bellocq}

L'invisibilisation et le déni de la mort dans les sociétés modernes sont des thèmes chers aux études funéraires ${ }^{1}$. Ces deux phénomènes prennent forme avec la disparition de la mort dans le quotidien des individus et notamment l'expulsion des cimetières à la périphérie des villes et des villages. Les mégapoles chinoises, et Shanghai en particulier, n'échappent pas à ce phénomène. L'invisibilisation de la mort y est favorisée non seulement par des programmes de rénovation urbaine à grande échelle mais aussi par une volonté politique forte, c'est-à-dire une réforme des pratiques funéraires qui a donné lieu à Shanghai, et dans d'autres villes et régions, à la généralisation de la crémation.

Depuis trois décennies, Shanghai connait des transformations importantes ${ }^{2}$. La ville s'est étendue au-delà des districts urbains d'origine; bourgs et villages présents sur le territoire de la municipalité sont progressivement absorbés par la ville. Les districts centraux connaissent depuis les années 2000 un renouvellement urbain accéléré, les maisons basses font place à des immeubles de plusieurs dizaines d'étages, les populations d'origine sont déplacées et les activités qui avaient cours dans ces quartiers disparaissent. La gentrification des districts centraux de Shanghai est à l'œuvre, et avec elle la physionomie de ces derniers change radicalement ${ }^{3}$. Les boutiques funéraires

1 Philippe Ariès, Essais sur l'histoire de la mort en Occident du Moyen Âge à nos jours, Paris, Points Seuil, 1975 ; Louis-Vincent Thomas, La mort en question. Traces de mort, mort des traces, Paris, L'Harmattan, 1991 ; Louis-Vincent Thomas, Anthropologie de la mort. Paris : Payot, 1994 ; Jean-Didier Urbain, L'archipel des morts. Cimetières et mémoire en Occident, Paris, Payot, 2005.

2 Thierry Sanjuan écrit au sujet des transformations de Shanghai : «En vingt ans, Shanghai a connu l'équivalent des aménagements haussmanniens, de la modernisation automobile et des restructurations postmodernes et contemporaines de Paris, des années 1850 à celles du début du XXI e siècle ". http://www.constructif.fr/bibliotheque/2010-6/shanghai-1-exemple-d-unemetropolisation-acceleree.html?item_id=3034.

3 Afin de rendre compte de l'ampleur de ces transformations urbaines, nous pouvons mentionner les chiffres émis par le Bureau national des statistiques et cités par He Shenjing : entre 1995 et 2005, 820448 familles ont été déplacées, 41,88 millions de mètres carrés de logements ont 
" traditionnelles " disparaissent ou se trouvent fortement standardisées, il est de plus en plus difficile de pratiquer le brûlage d'offrandes en papier destinées aux morts dans les zones résidentielles de la ville centre ${ }^{4}$. Les cortèges funéraires ne sont pas autorisés et les cérémonies se tiennent dans l'un des deux seuls gros centres funéraires des districts centraux. Il subsiste cependant encore une trace des cimetières dans la ville par le biais de bureaux qui les représentent. Cependant, avec leurs devantures qui rappellent celles d'agences immobilières, ces bureaux se fondent dans le paysage urbain.

Ces transformations urbaines contribuent largement à l'accélération de la mise en œuvre de la réforme funéraire adoptées dès la fin des années 1950 et sans cesse réaffirmée depuis. Celle-ci visait, dans un premier temps, à généraliser la crémation, à dépouiller les pratiques funéraires de tout élément associé à des "superstitions " ${ }^{5}$, à réduire le coût des funérailles en prônant notamment des cérémonies funéraires peu dispendieuses et simplifiées à l'extrême, l'objectif était de limiter le " gaspillage " de matières premières (bois pour le cercueil, pierre tombale, empreinte foncière) $)^{6}$.

Dans un contexte politique et urbain qui accorde si peu de visibilité à la mort ${ }^{7}$, nous nous interrogerons sur la place accordée aux cimetières aujourd'hui à Shanghai. Pour cela, dans une première partie, nous montrerons comment

été rasés. Shenjing He, «State-sponsored Gentrification Under Market Transition. The Case of Shanghai ", Urban Affairs Review, vol. 43, n² 2, nov. 2007, p. 77-78.

4 En diverses occasions (fêtes des morts, anniversaires, rupture du deuil, etc.), les Chinois rendent hommage à leurs morts en brûlant des offrandes en papier pouvant représenter de l'argent ou tout autre objet du quotidien car les morts dans l'autre monde ont des besoins similaires à ceux des vivants. Ces offrandes s'inscrivent dans le culte des ancêtres, une des expressions de la piété filiale. Un culte mal rendu peut avoir des conséquences fâcheuses sur les descendants vivants.

5 La notion de superstitions en Chine est opposée à celle de "science » et désigne les croyances qui n'appartiennent pas aux grandes religions. Ces croyances populaires et les pratiques qui vont avec sont prises pour cible par la réforme funéraire. Il n'est cependant pas toujours aisé de distinguer religion et superstition : "Les législateurs et les fonctionnaires, dès 1912 et encore aujourd'hui, doivent donc s'atteler à une tâche complexe : démêler la religion de la superstition, entreprise ardue dans la mesure où ces notions ne correspondent à aucune catégorie proprement chinoise, mais cruciale puisqu'elle dicte concrètement la politique religieuse sur le terrain, les autorisations ou interdictions de fêtes et rituels, la protection ou la destruction des temples. Les scientifiques sont parfois convoqués par le pouvoir pour aider à ce travail, et encore aujourd'hui cela fait partie des attributions des chercheurs en sciences des religions en République Populaire. " Vincent Goossaert, "Le concept de religion en Chine et l'Occident », Diogène, 205, 2004, p. 14.

6 Lin Fang, Vincent Goossaert (2008), "Les réformes funéraires et la politique religieuse de l'État chinois, 1900-2008 ", Archives de Sciences Sociales des Religions, 144, 2008, p. 51-73; Martin K. Whyte (1988), "Death in the People's Republic of China ", James L. Watson, Evelyn S. Rawski (éds), Death Ritual in Late Imperial and Modern China, Berkeley, University of California Press, 1988, p. 289-316.

7 Dans cet article, il sera exclusivement question de la mort dans l'espace public et plus particulièrement des cimetières. Mais la mort est également présente au domicile, par les rituels qui peuvent y être effectués, et au sein des temples par le biais de cérémonies commémoratives ou de salles dans lesquelles sont disposées des tablettes commémoratives individuelles ou conjugales. 
les cimetières depuis qu'ils existent à Shanghai ont été maintenus à distance, nous verrons également que les problèmes qu'ils posent aujourd'hui sont les mêmes depuis un siècle. Dans une deuxième partie, nous verrons comment, pour la municipalité de Shanghai, le numérique pourrait devenir une alternative sérieuse aux cimetières mais aussi un moyen d'aller plus dans la réforme des pratiques funéraires et de mieux contrôler ces dernières.

Cet article s'appuie sur des études de terrain réalisées à Shanghai entre 2009 et aujourd'hui durant lesquelles je me suis rendue dans 14 cimetières de la municipalité afin de procéder à des observations et à des entretiens auprès des usagers, des employés et de la direction lorsque cela était possible. Lors de mes études de terrain, je me suis également intéressée aux deux centres funéraires centraux, Xibaoxing et Longhua, auprès desquels j'ai eu l'opportunité de réaliser de longues sessions d'observation ainsi qu'une série d'entretiens. Ces dernières années, mes enquêtes de terrain ont davantage porté sur les boutiques funéraires ${ }^{8}$.

\section{Des cimetières dont on ne sait que faire / Des cimetières bien encombrants / Des cimetières sous pression}

Si les cimetières ont définitivement quitté la ville-centre de Shanghai à la fin des années $1950^{\circ}$ et qu'aujourd'hui la mort est à peine perceptible en ville, la mise à distance des morts n’a pas toujours été aussi marquée spatialement ${ }^{10}$. Jusqu'en 1912 la ville de Shanghai était ceinte par une muraille à l'intérieur de laquelle la population dense rendait difficile l'aménagement de lieux d'inhumation à l'intérieur de ces murs. Cependant, les riches familles n'hésitaient pas à ériger des tombes à l'intérieur même de la ville ${ }^{11}$. Comme le montre Christian Henriot ${ }^{12}$, il existait alors trois types de lieux de sépultures : les

8 Ces études de terrain ont dans un premier temps été financées et réalisées dans le cadre du programme ANR FunérAsie.

9 Seul le cimetière des martyrs et des héros de la Révolution se trouve dans la ville-centre. Les martyrs de la Révolution, les figures du parti communiste ont le privilège de pouvoir y reposer ; les citoyens ordinaires, une fois décédés, doivent eux quitter la ville et peupler les cimetières de la périphérie.

10 En effet, jusqu'au début du XXe siècle, une fois le cercueil scellé, la dépouille pouvait rester un temps, qui se comptait en mois voire en années, à proximité des vivants en signe de respect à l'égard du défunt: "When the coffin had been sealed, it was ready for removal from the village, town, or neighborhood of the deceased. This expulsion was the last formal act in the sequence of funerary rites, but it need not be accomplished immediatly. In fact, high status families (including the imperial household [...]) oftend kept the coffin in the domestic realm for months-even years-as mark of respect for the deceased. But, in the end, the coffin must be expelled from the domain of the living. ", James L. Watson, p. 15.

11 Christian Henriot, Scythe and the City. A Social History of Death in Shanghai, Stanford, Stanford University Press, 2016, p. 143.

12 Ibid., p. 145. 
tombes individuelles, les cimetières familiaux, les cimetières de charité et des cimetières réservées à des guildes. L'ensemble était généralement situé dans la campagne environnant la ville fortifiée. Il arrivait que dans l'attente de la décision d'un spécialiste du fengshui ou d'un maître taoïste, quant au lieu et la date à laquelle il fallait procéder à l'inhumation, la dépouille scellée dans son cercueil soit stockée dans un temple ou placée dans un champ, hors-terre pendant plusieurs années ou même plusieurs décennies ${ }^{13}$. Il n'existait alors pas de réglementation stricte ni de lieu spécifique où faire reposer les morts.

Dès les années 1920, ces tombes sauvages et ces cercueils hors-sol devinrent la cible de critiques : des terres cultivables sont ainsi occupées, des questions d'ordre sanitaire se posent également ${ }^{14}$. C'est alors que se développent des cimetières privés et publics dans le cadre de la promotion d'un traitement des morts mieux adapté à la vie moderne. Ces cimetières et en particulier ceux qui étaient publics ont souffert pendant un temps de l'image négative qui pesait sur les cimetières de charité, ancêtres du cimetière moderne ${ }^{15}$. Les familles aisées étaient peu enclines à enterrer leurs morts dans un cimetière public où reposaient des personnes avec un statut social inférieur au leur. Tout au long de la première moitié du XX $\mathrm{XX}^{\mathrm{e}}$ siècle, au fur et à mesure de l'expansion urbaine et de la proximité des vivants qu'elle entrainait, tombes et cimetières sont déplacés.

À l'arrivée des communistes au pouvoir en 1949, afin de remédier au manque d'espace disponible dans les cimetières, la municipalité de Shanghai encourage la pratique de la crémation ${ }^{16}$ avant que celle-ci ne soit imposée lors de la Révolution culturelle en raison des saccages commis par les Gardes rouges dans les cimetières ${ }^{17}$. À partir des années 1980, la municipalité s'engage à étendre certains cimetières existants et à en aménager de nouveaux dans des zones situées très à l'extérieur de la ville, et cela en ayant parfois recours à des capitaux privés ${ }^{18}$. Il s'agit pour ces nouveaux cimetières non pas d'accueillir des cercueils mais des coffrets cinéraires qui seront placés sous une pierre tombale. Conformément aux objectifs affichés par la réforme funéraire, les ressources naturelles (foncier, matières premières) sont ainsi en partie préservées.

Dès les années 2000, les spécialistes et acteurs du secteur funéraire de Shanghai estiment que la municipalité, après plusieurs décennies d'efforts, a achevé le premier volet de la réforme funéraire, centré sur la généralisation de

13 Ibid., p. 146-147. Il s'agissait de champs dans lesquels des morts avaient déjà été inhumés.

14 Ibid., p. 144.

15 Il s'agit de cimetières aménagés à la périphérie de la ville fortifiée de Shanghai qui étaient destinés aux plus pauvres ou cadavres non réclamés. Ibid., p. 152-170.

16 Avant l'arrivée des communistes au pouvoir des tentatives de promotion de la crémation ont vu le jour sans grand succès.

17 Christian Henriot, Scythe and the City. A Social History of Death in Shanghai, op. cit., p. 178-192.

18 Natacha Aveline, "La renaissance de l'industrie funéraire à Shanghai, cas exemplaire pour la Chine ", in Natacha Aveline (éd.), La place de morts dans les mégapoles d'Asie orientale, Paris, Les Indes savantes, 2013, p. 95. 
la crémation ${ }^{19}$. Ce premier volet qui reposait sur une volonté de préserver les ressources naturelles et le foncier a pu être accompli grâce à un long travail d'information voire de propagande, aux excès de la Révolution culturelle $e^{20}$, et par la suite, grâce à des investissements qui ont permis la mise en place d'un réseau de centres funéraires et de crématoriums. Dès les années 1980, le taux d'incinération atteint $95 \%$ à Shanghai ; aujourd'hui ce taux s'approche des $100 \%$, l'inhumation étant toujours autorisée pour les membres de la minorité musulmane Hui.

Après s'être heurtée au manque d'engouement des Shanghaiens pour la crémation, la poursuite de la réforme funéraire, qui consiste à minimiser l'empreinte foncière des cimetières et des tombes voire à les faire disparaittre, fait face à un nouvel obstacle : la place que les Shanghaiens accordent à la tombe dans le rapport qu'ils entretiennent avec leurs morts. Bien que la crémation soit désormais totalement passée dans les mœurs, la conservation des cendres, de préférence sous une tombe, reste profondément ancrée dans les habitudes des Shanghaiens. Le prix des tombes maintenu volontairement élevé ne les incite pas à renoncer à ce choix, tout comme la distance qui sépare les cimetières des districts centraux ${ }^{21}$.

Malgré cette persistance de l'usage des tombes, la crémation aurait cependant permis de réaliser, entre 1985 et 2006, une économie de 400 hectares sur le foncier. Mais, aujourd'hui, de nouvelles difficultés apparaissent avec des cimetières proches de la saturation, une situation qui n'est pas sans rappeler celle des années 1950. En effet, la pression démographique pèse fortement sur les espaces funéraires, le nombre de morts annuel à Shanghai est passé de 86000 en 1990 à 124000 en $2015^{22}$. Avec le vieillissement de la population, dans les années 2030 on comptera en moyenne 170000 morts par an et 240000 dans les années $2050^{23}$. Or, d'après des estimations avancées par le Quotidien du Peuple, en

19 Qiugen Gu, Kuanyuan Qiao, Shaoyu Zhou (éds), Gongmu Guanli Yanjiu Xin Lun, Shanghai, Shanghai Daxue Chubanshe, 2003 ; Qiugen Gu, "Binzang Xisu Gaige Yao Yushi Jujin », in Jinlong Zhu (éd.), Binzang Gaige he Wenhua jianshe Chutan, Shanghai, Shanghai Daxue Chubanshe, 2004, p. 308-313 ; Wei Wang, "Zhanshi Gongzuo, Jiji Tuijin Binzang Xisu de "Di-er Ci Geming" ", in Jinlong Zhu (éd.), Binzang Gaige heWenhua jianshe Chutan, op. cit., p. 352-355.

20 Christian Henriot, Scythe and the City. A Social History of Death in Shanghai, op. cit., p. 309-338 ; Vincent Goossaert, Ling Fang, «Les réformes funéraires et la politique religieuse de l'État chinois, 1900-2008 ", Archives de Sciences Sociales des Religions, 144, 2008, p. 59.

21 En Europe et notamment en France, un phénomène similaire peut être observé ; les cimetières sont peu et de moins en moins fréquentés et pourtant, l'habitude d'engager des frais importants pour l'achat d'une tombe persiste. Voir Jean-Didier Urbain, L'archipel des morts. Cimetières et mémoire en Occident, op. cit., p. 269.

22 http://www.stats-sh.gov.cn.

23 Qiugen Gu, Kuanyuan Qiao, Shaoyu Zhou (éds), Gongmu Guanli Yanjiu Xin Lun, op. cit., p. 31. 
2014, seule une centaine d'hectares, restaient disponibles dans les 44 cimetières de la municipalité d'une superficie totale de 500 hectares. La pénurie est plus ou moins importante selon les cimetières, mais d'après Gao Jianhua, responsable du bureau des affaires funéraires de Shanghai, plus des trois quarts des cimetières de Shanghai se trouvent dans une situation proche de la saturation et une bonne partie d'entre eux n'ont pas la possibilité de s'étendre ${ }^{24}$. Le secteur funéraire de la municipalité tente de répondre à ces contraintes par la promotion depuis deux décennies de nouveaux modes de sépulture parmi lesquels des tombes d'une superficie inférieure à $1 \mathrm{~m}^{225} 26$.

Les problèmes posés par la quasi-saturation des cimetières à Shanghai ne sont cependant pas résolus et les autorités encouragent ces derniers à pratiquer la dispersion des cendres se trouvant sous les tombes les plus anciennes et dont les familles ne s'acquittent plus des charges annuelles ou décennales. Bien que couplés à une volonté de réduire la durée des concessions, ces dispositifs restent largement insuffisants pour contrer la pression foncière. Les cimetières sont alors contraints de se verticaliser et les tombes de se miniaturiser. Mais sans l'aménagement d'espaces supplémentaires, ces stratégies ne suffiront pas à faire face au vieillissement de la population, synonyme dans les cimetières de croissance démographique. Ainsi, les autorités funéraires de Shanghai souhaiteraient pousser la réforme plus loin et parvenir à la non-conservation des restes et échapper ainsi aux contraintes spatiales. À cette fin, la municipalité de Shanghai encourage la dispersion des cendres en mer et tente de développer un cimetière en ligne.

\section{Vers une mort et des rituels dématérialisés...}

Malgré quelques ajustements réglementaires visant à réduire l'empreinte foncière des tombes, la pression sur les cimetières reste forte si bien que l'inhumation des cendres et les nouveaux modes de sépultures économes en foncier sont présentés par la municipalité comme des procédés transitoires devant conduire à la seconde phase de la réforme funéraire dont l'objectif est la nonconservation des restes ${ }^{27}$.

24 http://finance.sina.com.cn/china/20140404/060918709389.shtml.

25 Qiugen Gu, Kuanyuan Qiao, Shaoyu Zhou (éds), Gongmu Guanli Yanjiu Xin Lun, op. cit., p. 35 ; Natacha Aveline, "La renaissance de l'industrie funéraire à Shanghai, cas exemplaire pour la Chine ", op. cit., p. 89.

26 L'article 16 du Règlement pour la gestion des cimetières de Shanghai (Shanghai Shi Gongmu guanli banfa) interdit la vente de tombes individuelles d'une superficie supérieure à $1,5 \mathrm{~m}^{2}$ et de tombes doubles d'une superficie supérieure à $3 \mathrm{~m}^{2}$. http://www.shanghai.gov.cn/shanghai/ node2314/node2319/n31973/n32004/n32016/n32018/u21ai858089.shtml.

27 Qiugen Gu, Kuanyuan Qiao, Shaoyu Zhou (éds), Gongmu Guanli Yanjiu Xin Lun, op. cit., p. 9-10, 29-37, 233 ; Qiugen Gu, "Binzang Xisu Gaige Yao Yushi Jujin », in Jinlong Zhu (éd.), Binzang Gaige he Wenhua jianshe Chutan, op. cit., p. 308-313. 
Lorsqu'ils traitent de cette seconde phase qu'ils qualifient de "deuxième révolution funéraire ", $\mathrm{Gu}^{28}$ et Wang ${ }^{29}$ se réfèrent aux réflexions de Zhou Enlai ${ }^{30}$. Le passage de la conservation du corps à la non-conservation de celui-ci, soit la généralisation de la crémation, constituait la première " révolution " funéraire alors que le passage de la conservation des cendres à leur non-conservation devrait constituer une deuxième "révolution" funéraire plus radicale encore ${ }^{31}$.

À cette fin, la municipalité de Shanghai a commencé à promouvoir les dispersions de cendres en mer (海葬 haizang, sépulture maritime) en 1991, qui se font de manière contrôlée et collective lors de rassemblements sur des bateaux affrétés par le Funeral Interment Service (FIS) ${ }^{32}$. Entre 1991 et fin 2017, le contenu de 40520 urnes cinéraires a ainsi été dispersé en mer et le contenu de 3000 urnes pour la seule année 2016. Ces chiffres paraissent encore extrêmement limités au regard du nombre de morts annuel à Shanghai mais semble en progression. Il en est de même du regard des Shanghaiens sur les "sépultures maritimes ". D’après une enquête de 2016 réalisée par l'université Ligong, 24,7\% des Shanghaiens se montreraient favorables aux sépultures maritimes contre $4 \%$ seulement en $2003^{33}$.

Afin d'encourager la dispersion des cendres en mer, la municipalité de Shanghai va plus loin et a mis en place, à partir de 1999, un système de subventions destinées aux familles. En 1999, 150 yuans étaient accordés aux

28 Qiugen Gu, Kuanyuan Qiao, Shaoyu Zhou (éds), Gongmu Guanli Yanjiu Xin Lun, op. cit. ; Qiugen Gu, "Binzang Xisu Gaige Yao Yushi Jujin », in Jinlong Zhu (éd.), Binzang Gaige he Wenhua jianshe Chutan, op. cit.

29 Wang Wei est le chef adjoint du Bureau des Affaires civiles de Shanghai. Wei Wang, "Zhanshi Gongzuo, Jiji Tuijin Binzang Xisu de "Di-er Ci Geming" ", in Jinlong Zhu (éd.), Binzang Gaige heWenhua jianshe Chutan, op. cit.

30 Ces deux auteurs ne mentionnent pas de références précises au sujet des réflexions de Zhou Enlai. Mais, sur le site d'information du Parti communiste chinois un article a été publié dans lequel il est question des réflexions de Zhou Enlai au sujet de la crémation et de la non conservation des restes. «Lieu et signification de la dispersion des cendres de Zhou Enlai » (Zhou Enlai guhui sanluo didian yu hanyi). sahttp://dangshi.people.com.cn/n/2015/0921/c85037-27611288.html.

31 La généralisation de la crémation des défunts dans un laps de temps finalement assez limité peut effectivement être considérée comme une révolution dans la mesure où la crémation revenait à enfreindre un précepte confucianiste selon lequel le maintien du corps du défunt dans son intégrité constituait un acte de piété filiale pour ses descendants.

32 Funeral Interment Service est un opérateur public sous l'autorité du bureau des Affaires civiles de Shanghai. Voir Natacha Aveline, "La renaissance de l'industrie funéraire à Shanghai, cas exemplaire pour la Chine", op. cit., p. 85-86.

33 Voir les articles de The Paper et de Chinanews sur le sujet : "À Shanghai il n'est possible de disperser en mer les cendres des défunts que trois mois par an, le temps d'attente est en moyenne de six mois " (Shanghai mei nian jin san ge yue ke haizang pingjun dengdai shijian wei bannian zouyou). http://sh.sina.com.cn/news/m/2018-03-24/detail-ifysnevm6710224.shtml ; " À Shanghai les cendres de plus de 37000 défunts ont été dispersées en mer, une proportion en hausse chaque année " (Shanghai yu 3.7 wan ming shizhe " guhui sanhai " haizang zhanbi zhunian pansheng). http://www.sh.chinanews.com/spxw/2017-03-26/20494.shtml. 
familles qui faisaient le choix d'une sépulture maritime pour l'un de leurs proches, puis 400 yuans en 2007, 2000 yuans en 2012 et 4600 yuans depuis 2018. Aujourd'hui, sur ces 4800 yuans, 3000 yuans sont directement remis à la famille et les 1600 yuans restants sont destinés à l'organisation de la dispersion des cendres sur un bateau affrété par le FIS ${ }^{34}$.

Une des limites des sépultures maritimes réside dans le fait que, une fois les cendres dispersées, en dehors d'un certificat attestant de leur dispersion et de la gravure du nom du défunt sur la stèle collective du cimetières Binhai Guyuan, il ne reste rien aux familles, elles ne disposent d'aucun endroit où se recueillir ou déposer des offrandes au moment de la fête des morts ou en d'autres occasions. Vu la faible proportion que représente la dispersion des cendres en mer, la grande stèle érigée dans le cimetière Binhai Guyuan ne suffit pas à lever toutes les résistances à l'égard de ce mode de sépultures. À partir des années 2000, les textes circulant au sein du secteur funéraire commencent à mentionner le potentiel que pourrait représenter internet pour assurer un support mémoriel qui se substituerait aux tombes une fois les cendres dispersées ou qui viendrait compléter les autres formes de sépultures. Dans ces textes, il est souligné l'espace illimité que propose internet par opposition à l'espace limité dont dispose la municipalitée ${ }^{35}$.

Dans cette perspective, depuis mars 2000, le FIS propose sur son site un cimetière virtuel. La particularité de ce cimetière est d'être issu d'une initiative institutionnelle contrairement à d'autres cimetières du même type qui peuvent avoir été créés par des entreprises spécialisées, des associations ou même par de "vrais" cimetières qui proposent sur le net un prolongement de leurs services ${ }^{36}$. À Shanghai, les familles dont les cendres d'un proche ont été dispersées en mer ont désormais la possibilité de créer une tombe virtuelle sur le site du FIS et, en principe, de compenser ainsi l'absence de lieu où se recueillir en dehors de la stèle collective érigée au sein du cimetière Binhai Guyuan.

La page d'accueil de ce cimetière en ligne propose un moteur de recherche qui permet de retrouver la " tombe " d'un proche. Elle présente également des vignettes avec la photo et le nom de différentes personnes qui ont leur

34 Voir article du Dongfan Zaobao mis en ligne en décembre 2012 sur le site du Bureau des Affaires civiles de Shanghai, "Shanghai augmente ses subventions afin de répondre aux obstacles rencontrés par les sépultures maritimes ", Shencheng tisheng butie huanjie haizang nanti ». http://www.shmzj.gov.cn/gb/shmzj/node4/node13/node1562/u1ai34623.html.

35 Shanghai Binzang Wenhua Yanjiusuo, Ha'erbin Huangshan Gongmu, Xin Shiji Gongmu Fazhan Zhanlüe Xueshu Yanjiuhui, recueil de contributions. Document interne, non daté, p. $445-452$.

36 " "Cimetières virtuels" et "cimetières en ligne" " (2009) ("Shangwan gongmu" yu "gongmu shangwang"), http://www.wangzang.cn/Newsindex.asp?id=418; Hélène Bourdeloie, H. (2015). "Usages des dispositifs socionumériques et communication avec les morts. D'une reconfiguration des rites funéraires ", Question de communication, 28, 2005, p. 3 ; Fanny Georges, "Identité post mortem et nouvelles pratiques mémoriales en ligne. L'identité du créateur de la page mémoriale sur Facebook ", Les Cahiers du Gerse, 2015, p. 55. 
sépulture sur le site. Un clic sur une de ces vignettes fait apparaitre la tombe virtuelle du défunt, un onglet permet alors d'accéder à une présentation du défunt, de ses qualités et des événements qui ont marqué sa vie. Un autre onglet nous conduit vers une page sur laquelle peuvent figurer des photos du mort, jusqu'à une dizaine, à divers âges. Enfin, un troisième onglet permet d'accéder à une page sur laquelle il est possible de procéder à des offrandes virtuelles : bouquets de fleurs, morceaux de musique, alcools.

Lorsque les proches effectuent des offrandes en ligne, ils ont la possibilité de laisser un message écrit, daté et pour lequel ils doivent préciser leur lien avec le mort. Ces messages sont souvent l'occasion de donner des nouvelles de la famille, de la santé de ses membres, d'annoncer une naissance, un changement de situation professionnelle, etc. Dans certains d'entre eux sont également décrits les funérailles, le déroulement de la fête des morts Qingming, les offrandes qui ont été brûlées pour l'occasion, etc. Les dates mentionnées en tête de messages montrent que la plupart des offrandes virtuelles sont réalisées lors d'événements justifiant une visite sur la tombe : la fête des morts, le solstice d'hiver, l'anniversaire de la mort ou de la naissance, etc.

Ces petits textes qui accompagnent les offrandes virtuelles et qui souvent décrivent les offrandes rituelles effectuées au domicile montrent que les pratiques traditionnelles de brûlage d'objets de papier votifs perdurent. La tombe virtuelle n'annule pas nécessairement les pratiques anciennes, elle aurait même plutôt tendance à les compléter. Fiorenza Gamba ${ }^{37}$ définit les rituels numériques comme une "ritualité parallèle ". Mais, dans le cas du cimetière virtuel mis en ligne par la municipalité de Shanghai, il s'agit à la fois d'une ritualité de substitution, dans la mesure où ce cimetière a été mis en place afin de suppléer les nécropoles matérielles, et d'une " ritualité parallèle " dès lors que les rituels qui se doivent d'être conduits sur la tombe le sont au domicile sous leur forme traditionnelle, et en ligne sous la forme d'un clic sur une icône.

Cependant, il est intéressant de noter qu'aucune offrande traditionnelle comme la monnaie de papier ni même de l'encens n'est proposée. Le site préfère mettre à disposition des offrandes qui répondent aux objectifs de la réforme funéraire en matière de contrôle des pratiques funéraires et du culte des morts. En effet, l'article 36 du Règlement pour la gestion des affaires funéraires (2018) stipule que la fabrication et la vente d'" objets funéraires superstitieux " sont interdites ${ }^{38}$. D'autres textes qui circulent au sein du secteur funéraire de Shanghai préconisent un recours à la "science " et aux « sciences humaines " pour réformer « les pratiques funéraires transmises depuis plusieurs millénaires ${ }^{39}$. Les cimetières internet sont d'ailleurs présentés comme un

37 Fiorenza Gamba, Mémoire et immortalité au temps du numérique. L'enjeu de nouveaux rituels de commémoration, Paris, L'Harmattan, 2016, p. 54.

38 http://www.law-lib.com/fzdt/newshtml/20/20180908094518.htm.

39 Qiugen Gu, Kuanyuan Qiao, Shaoyu Zhou (éds), Gongmu Guanli Yanjiu Xin Lun, op. cit., p. 3. 
moyen d'éliminer progressivement " les mauvaises habitudes funéraires traditionnelles $»^{40}$. Ainsi, dans le cimetière en ligne de Shanghai, l'encens et tous les articles votifs habituellement représentés en papier et destinés à être brûlés (argent, maison, voiture, etc.) sont totalement absents des offrandes proposées. Finalement, le cimetière internet permet d'aller plus loin dans la réforme funéraire en facilitant, en principe, la non conservation des restes et en visant indirectement les pratiques funéraires associées au culte des ancêtres et liées, selon la phraséologie du Parti communiste chinois, à des "superstitions".

Un parallèle peut être établi entre les pratiques liées au culte des morts dans la vie réelle et celles proposées sur internet. Dans les pratiques réelles, la mise à feu, la fumée permettent de transmettre au défunt les paroles et les objets votifs en papier. Sur internet, le clic qui peut s'apparenter à une mise à feu permet aux messages et aux offrandes d'être transmis aux morts dans l'autre monde, le monde des morts, le monde virtuel, le cloud (yun, nuage). Au niveau des représentations, cela semble pouvoir fonctionner. D’ailleurs, certains promoteurs des cimetières virtuels en Chine vont jusqu'à évoquer la "magie " de la souris et du clic lorsque l'on fait des offrandes en ligne, et une existence numérique qui permettrait aux morts de devenir immortels ${ }^{41}$.

\section{La crise sanitaire de la covid-19 et les « télérituels »}

La crise sanitaire liée à l'épidémie de covid pourrait bien donner un coup d'accélérateur au développement des cimetières et des rituels funéraires en ligne. Tous les ans début avril, les Chinois célèbrent la fête des morts Qingming. Il s'agit d'un moment important dans la relation que les Chinois entretiennent avec leurs proches disparus, qui donne lieu à une surfréquentation des cimetières. Les membres de la famille se rassemblent devant la tombe de leurs proches disparus : ils la nettoient, y déposent un service d'offrandes, font brûler de la monnaie de papier puis, à tour de rôle, chacun des membres de la famille avec des bâtons d'encens dans les mains jointes s'inclinera trois fois devant la sépulture. Les membres de la famille profitent de l'occasion pour se retrouver, c'est également un prétexte pour faire acte de piété filiale à l'égard de ses parents décédés ${ }^{42}$. La fête des morts est aussi un jour privilégié pour la mise en terre des coffrets cinéraires qui se trouvaient jusque-là conservés dans un columbarium du centre funéraire.

40 Shanghai Binzang Wenhua Yanjiusuo, Ha'erbin Huangshan Gongmu, Xin Shiji Gongmu Fazhan Zhanlüe Xueshu Yanjiuhui, op. cit., p. 449.

41 Ibid., p. 447.

42 La piété filiale est une valeur centrale à l'organisation familiale chinoise, elle régit les relations entre enfants et parents et cela même après la mort de ces derniers. Le culte des ancêtres est un acte de piété filiale. 
Cette année, la crise sanitaire lié à la Covid-19 a quelque peu perturbé la célébration de la fête des morts. Un document publié par la direction générale du ministère des Affaires civiles ${ }^{43}$ invitait les gouvernements locaux à prendre des mesures afin de limiter la propagation du virus lors de ces festivités. En fonction de la situation locale, il s'agissait de fermer l'accès aux cimetières ou d'en limiter l'accès afin d'éviter des rassemblements trop importants. Il revenait aux cimetières d'adapter et d'organiser les flux en leur sein ou de proposer une alternative. Le document n’omet pas de rappeler la nécessité de promouvoir des commémorations et de nouvelles pratiques " écologiques »; cela passe par le développement actif des services funéraires en ligne et notamment pour les offrandes.

Suite à ce texte du ministère des Affaires civiles, la municipalité de Shanghai a présenté des mesures à mettre en œuvre lors de la fête des morts ${ }^{44}$. La municipalité de Shanghai, bien qu'officiellement peu touchée par l'épidémie de coronavirus $^{45}$, a préconisé des commémorations à distance. Il était toutefois possible de se rendre sur place dans des créneaux horaires précis à condition de s'être inscrit préalablement en ligne. Le document publié par la municipalité rappelle qu'il est interdit de brûler des offrandes en papier, qualifiés d'articles superstitieux, dans l'enceinte des cimetières ou de faire exploser des pétards, afin de "poursuivre la réforme funéraire et d'établir de nouvelles habitudes".

Suite à ces consignes, les cimetières de la ville ont pu mettre en place des services de commémoration de Qingming en ligne ${ }^{46}$. C'est le cas, par exemple, du très chic cimetière Fushou ${ }^{47}$ qui propose un service intitulé "Yun (le nuage/ le cloud) Fushou " grâce auquel il est possible de retrouver la tombe en ligne d'un proche et d'effectuer des offrandes selon des modalités similaires à celles du cimetière décrit précédemment. Les proches peuvent choisir un type d'offrandes (bouquet de fleurs, bougies, encens, alcool blanc, fruits, cigarettes, etc.) qui n'est pas officiellement associé à des « superstitions $»^{48}$.

43 民政部办公厅关于做好2020年清明节祭扫工作的通知, 13 mars 2020. http://www.gov. cn/zhengce/zhengceku/2020-03/15/content_5491507.htm.

44 Shanghai par le document 关于做好上海市 2020 年清明期间预约祭扫工作的通知, élaboré par le Bureau en charge de la fête Qingming. 关于做好上海市2020年清明期间预约祭扫工作的通 知沪清明办, 19 mars 2020. http://mzj.sh.gov.cn/gb/shmzj/node8/node194/u1ai47911.html.

45 Officiellement, le 26 juin 2020, Shanghai recensait au total 705 cas de covid-19 et 7 décès. https://gisanddata.maps.arcgis.com/apps/opsdashboard/index.html\#/bda7594740fd40299423467b48e9ecf6.

46 Les cimetières ont également mis en place des commémorations collectives ou individuelles pratiquées sur demande par les employés afin de compenser l'absence des proches restés contraints de rester chez eux.

47 http://www.k366.com/rili/100489.html.

48 Lors d'une prochaine enquête de terrain, il sera nécessaire d'appréhender l'impact que la fête de Qingming de 2020 aura eu sur les pratiques de commémorations des morts. 


\title{
Conclusion
}

Lorsque l'on observe l'évolution des cimetières à Shanghai, on constate que l'emprise matérielle de la mort semble destinée à être toujours plus réduite voire même à perdre son empreinte spatiale. Il s'agit pour la municipalité de répondre à des contraintes urbaines mais également à une volonté politique forte des autorités funéraires. L'objectif est de développer la ville sans entraves et de poursuivre la réforme des pratiques funéraires : aboutir à la non-conservation des restes et développer des rituels d'offrandes qui ne soient pas liées à des "superstitions ». Le développement des sépultures maritimes devrait permettre à terme de répondre à ce double objectif car, en effet, les cimetières en ligne présentent l'avantage d'être plus qu'économes en foncier et de mieux contrôler les pratiques en ne proposant que des offrandes qualifiées de "civilisées" (wenming).

Aujourd'hui, les sépultures maritimes ne sont pas très populaires et ne représentent qu'une faible proportion des sépultures chaque année car elles entrent en contradiction avec les principes de la piété filiale. Mais leur généralisation est une hypothèse qu'il ne faut pas pour autant négliger. La Chine a montré par le passé qu'elle était capable de mettre en œuvre des mesures très impopulaire et en contradiction avec la tradition. Selon les principes confucianistes il convient de conserver la dépouille d'un proche dans son intégrité et cela constitue un acte de piété filiale ; or, la crémation, malgré de fortes réticences, a été imposée, généralisée à Shanghai en à peine deux décennies. La politique de l'enfant unique est une autre mesure qui a brisé un des fondements de la famille traditionnelle chinoise.

Ces nouvelles pratiques virtuelles que la municipalité de Shanghai tente de diffuser permettent également de répondre à de nouveaux modes de vie marqués par la mobilité géographique. Cette "deuxième révolution funéraire ", qui vise à repousser les cimetières toujours plus loin et qui cherche à combattre certaines représentations et pratiques funéraires, contribue à un certain renouvellement des rituels et, peut-être aussi, comme l'écrit Gamba, à " une dissolution des rituels dans des activités quotidiennes ${ }^{49}$. Le cimetière virtuel est en effet accessible à tout moment et en tout lieu. Ainsi, à force d'avoir été repoussé, le cimetière finira, peut-être, par occuper une place centrale malgré son invisibilisation.

\author{
Maylis Bellocq \\ TELEM EA 4195 / ECMC-EHESS \\ Université Bordeaux Montaigne \\ Maylis.Bellocq@u-bordeaux-montaigne.fr
}

49 Fiorenza Gamba, Mémoire et immortalité au temps du numérique. L'enjeu de nouveaux rituels de commémoration, op. cit., p. 69. 


\section{Résumé}

Dans cet article nous nous interrogeons sur la place accordée aux cimetières aujourd'hui à Shanghai. Dans un contexte de forte pression foncière, l'expansion urbaine et le vieillissement de la population font peser de lourdes contraintes sur les cimetières de Shanghai qui n’ont plus la possibilité de s'étendre. Après avoir montré comment la municipalité a toujours veillé à maintenir ses cimetières à distance, nous analysons la manière dont ils sont amenés à se réinventer. Nous voyons également comment internet offre de nouvelles perspectives aux cimetières de la ville en proposant un nouveau type de pratiques funéraires.

\section{Mots-clés}

Cimetières, Shanghai, internet, réforme funéraire, rituel en ligne.

\section{Abstract}

This article examines the place cemeteries occupy in present-day Shanghai. Amid pronounced land development, urban expansion and an ageing population, Shanghai's cemeteries are under severe pressure, not least their inability to expand any further. After demonstrating how municipal authorities have consistently undertaken measures to maintain a certain distance between the city and its cemeteries, analysis shifts to how these are currently being reinvented. In this latter regard, the article pays a particular attention to the ways in which the internet is providing new opportunities to Shanghai's cemeteries to offer new funerary practices.

\section{Keywords}

Cemeteries, Shanghai, internet, funerary reform, rituals on line. 